\title{
Diseño factorial en la investigación de la reactividad de los crudos de cemento
}

\author{
Factorial Design in Cement Raw Mix Reactivity \\ Investigation
}

\author{
GABRIEL LUIS DUQUE FERNÁNDEZ y JOSEFINA DE LAS MERCEDES CARDOSO CAMACHO \\ Laboratorio Central del Cemento \\ ESTER RUBIO FRÍAS, Centro de Estudios Aplicados al Desarrollo Nuclear \\ LA HABANA-CUBA \\ WILHEM MULLER, Wissenchaftsbereich Verfarenstechnik Fachbereich Bindebaustoffe
}

Fecha de recepción: 22-V-1989

\section{RESUMEN}

En el presente trabajo se corrobora que las técnicas de diseño de experimento son aplicables a las

investigaciones de facilidad de quema de los crudos de cemento, obteniéndose resultados que, abarcando el rango de concentraciones de interés industrial,

concuerdan de forma satisfactoria con lo esperado por la teoria, con considerable ahorro de tiempo y dinero.

Se encontró una interacción con signo negativo entre el factor de saturación de cal (FSC) y el módulo de silice,

tanto en facilidad de quema de crudos como en

reactividad del cemento.

Se recomienda trabajar con factor de saturación de ca! alto y módulo de silice bajo para el rango de concentraciones estudiado.

\author{
ABSTRACT \\ The present paper corroborates, that experimental design \\ technics are applicable to raw mix burnability \\ investigations, and with it the obtained results could cover \\ all the range of possible concentrations of industrial \\ interest. Obtained results agree with theory.
}

A negative interaction was found between lime saturation factor (LSF) and silicate module(Ms) in raw mix burnability' and in cement reactivity.

In the concentration range studied, working with high LSF and low Ms is recomended.

\section{INTRODUCCION}

De todas las etapas del proceso de producción del cemento la más costosa, desde el punto de vista energético, es la fabricación del clínker en el horno rotatorio; la misma consume unos $3.140 \mathrm{~kJ} / \mathrm{kg}$ de producto terminado [1].

La optimización de la reactividad de la mezcla de crudos es una de las vías para aumentar la productividad del horno, obteniendo ahorros sustanciales de energía.

Esta es la causa de que muchos autores realizaran investigaciones sobre el tema de reactividad de crudos y aditivos mineralizadores y (o) fundentes a los crudos del cemento $[2,3,4,5,6]$.

\section{INTRODUCTION}

The clinkerization of cement raw mixes, is the most costly stage in the production of this important construction product, needing more or less $3.140 \mathrm{~kJ} / \mathrm{kg}$ of finished product [1].

One of the ways to increase kiln productivity is the optimization of raw mix burnability, addition of fluxes and/or mineralizers [2, 3, 4, 5, 6]. 
Estos trabajos analizan los efectos del aditivo o de determinada mezcla con una composición fija pero, desde el punto de vista industrial, es necesario estudiar el comportamiento de estos aditivos en el rango de concentraciones en que fluctúan, el factor de saturación de cal (FSC), el módulo de sílice (Ms) y el módulo de alúmina (Ma) [7].

Henning [8] encontró que existía una correlación entre el espectro infrarrojo y el contenido de alita de las distintas fases del cemento.

Yamaguchi et al [9], propusieron el máximo de difracción que se encuentra, entre 2,715 y 2,885 angstroms, para evaluar el contenido de alita de un cemento.

En [7] se demostró que las técnicas de diseño de experimento son aplicables a este tipo de investigaciones, y el objetivo del presente trabajo es el estudio de la interrelación de las técnicas instrumentales con las técnicas de diseño de experimento.

\section{DESARROLLO DEL TRABAJO}

Se prepararon 6 muestras de crudo de alimentación al horno en los que se variaron los módulos y el FSC en el rango de trabajo de la fábrica, obteniéndose los resultados que se presentan en la tabla 1.
In this papers the effect of additives or some mix of fixed composition is analyzed, but in an industrial point of view, it is necesary a thorough study of all the range of concentrations in which the lime saturation factor (LSF), silicate and alumina modules of the investigated raw mix move [7].

Henning [8] found a correlation between the infrared espectra and the different phases content in cement.

Yamaguchi et al [9], proposed cement alite content evaluation through the difraction maximum at 2.715-2.885 angstroms.

In [7] it was demonstrated that this technics were applicable in this sort of investigation and the objectives of the present paper are the relationships between theory, instrumental technics and experimental design.

\section{EXPERIMENTAL PROCEDURE}

Six raw mixes were elaborated with different LSF, silicate module (Ms), and aluminate module (Ma), that cover the working range of the factory, obtaining the results shown in table 1.

TABLA 1 TABle 1

Composición química de las diferentes muestras (Chemical composition of the diferent samples)

\begin{tabular}{|c|c|c|c|c|c|c|c|c|c|}
\hline \multirow{2}{*}{$\begin{array}{l}\text { Muestra } \\
\text { (Sample) }\end{array}$} & \multicolumn{7}{|c|}{ Contenidos en \% en masa (Contents in mass \%) } & \multicolumn{2}{|c|}{$\begin{array}{l}\text { Parámetros de los } \\
\text { crudos (Parameters } \\
\text { of the raw mix) }\end{array}$} \\
\hline & $\mathrm{SiO}_{2}$ & $\mathrm{Al}_{2} \mathrm{O}_{3}$ & $\mathrm{Fe}_{2} \mathrm{O}_{3}$ & $\mathrm{CaO}$ & $\mathrm{MgO}$ & PPI & FSC & Ms & $\mathrm{Ma}$ \\
\hline 1 & 13,5 & 4,2 & 2,7 & 40,5 & 0,9 & 35,2 & 0,92 & 1,95 & 1,55 \\
\hline 2 & 13,2 & 4,1 & 2,6 & 40,7 & 1,2 & 35,2 & 0,94 & 1,94 & 1,58 \\
\hline 3 & 13,8 & 4,1 & 2,6 & 40,6 & 0,9 & 35,1 & 0,90 & 2,08 & 1,57 \\
\hline 4 & 13,2 & 3,9 & 2,5 & 41,0 & 0,8 & 35,3 & 0,95 & 2,06 & 1,57 \\
\hline 5 & 13,5 & 4,3 & 2,3 & 40,4 & 1,0 & 35,1 & 0,91 & 2,02 & 1,80 \\
\hline 6 & 13,1 & 4,0 & 2,3 & 41,3 & 0,9 & 35,7 & 0,94 & 1,99 & 1,72 \\
\hline
\end{tabular}

Las variables fueron codificadas, de tal forma [10], que con un subconjunto de cuatro de las seis muestras elaboradas se pudiera estudiar el efecto del FSC y el Ms (muestras 1, 2, 3 y 4), y con otro el efecto del FSC y el Ma (muestras $1,2,5$ y 6), con lo que se obtienen dos diseños factoriales $2^{2}$.
The variables were coded in such a way [10] that with a subset of four of the six samples, the effect of LSF and Ms could be studied (samples 1, 2, 3 and 4) and with the another the effect of LSF and Ma could be studied (samples 1, 2, 5 and 6). Then there are two $2^{2}$ factorial design experiments. 
Los valores de los parámetros para la codificación se muestran en la tabla 2.

Todas las muestras fueron calcinadas por el método descrito en [7], obteniéndose clínkeres cuya composición aparece en la tabla 3.
The coding values for the different parameters are shown in table 2.

All the samples were ignited by the method described in [7], obtaining clinkers with compositions shown in table 3.

TABLA 2 TABle 2

Codificación de las variables (Variables codification)

\begin{tabular}{|l|c|c|}
\hline Variable (Variable) & Valor menor (-1) [Lower value (-1)] & Valor mayor (+1) [Higher value $(+1)]$ \\
\hline FSC (LSF) & $0,90-0,92$ & $0,94-0,96$ \\
Ms & $1,94-2,02$ & $2,06-2,08$ \\
Ma & $1,55-1,58$ & $1,72-1,80$ \\
\hline
\end{tabular}

TABLA 3 TABLE 3

Composición química, mineralógica y contenido de fase líquida de los clínkeres

(Chemical, mineralogical and liquid phase contents of the studied clinkers)

\begin{tabular}{|c|c|c|c|c|c|c|}
\hline \multirow{2}{*}{$\begin{array}{c}\text { Muestra } \\
\text { (Sample) }\end{array}$} & \multicolumn{6}{|c|}{ Contenidos en \% en masa (Contents in mass \%) } \\
\cline { 2 - 7 } & $\mathrm{SiO}_{2}$ & $\mathrm{Al}_{2} \mathrm{O}_{3}$ & $\mathrm{Fe}_{2} \mathrm{O}_{3}$ & $\mathrm{CaO}$ & $\mathrm{MgO}$ & $\mathrm{Cl}$ \\
\hline 1 & 21,8 & 6,8 & 4,4 & 65,5 & 1.5 & 0,94 \\
2 & 21,5 & 6,6 & 4,2 & 65,9 & 1,9 & 0,91 \\
3 & 22,3 & 6,5 & 4,2 & 65,5 & 1,5 & 1,17 \\
4 & 21,5 & 6,4 & 4,1 & 66,7 & 1,3 & 1,47 \\
5 & 21,9 & 7,0 & 3,9 & 65,6 & 1,6 & 1,31 \\
6 & 21,3 & 6,5 & 3,8 & 67,0 & 1,4 & 1,11 \\
\hline
\end{tabular}

\begin{tabular}{|c|c|c|c|c|c|c|c|}
\hline \multirow[t]{2}{*}{$\begin{array}{l}\text { Muestra } \\
\text { (Sample) }\end{array}$} & \multicolumn{4}{|c|}{$\begin{array}{l}\text { Composición mineralógica según Bogue } \\
\text { (Bogue mineralogical contents) }\end{array}$} & \multicolumn{3}{|c|}{$\begin{array}{l}\text { Contenidos de fase líquida } \\
\text { a la temperatura de } \\
\text { (Liquid phase contents } \\
\text { at the temperature of) }\end{array}$} \\
\hline & $\mathrm{C}_{3} \mathrm{~S}$ & $\mathrm{C}_{2} \mathrm{~S}$ & $\mathrm{C}_{3} \mathrm{~A}$ & $\mathrm{C}_{4} \mathrm{AF}$ & 1.340 & 1.400 & 1.500 \\
\hline 1 & 45,1 & 28,5 & 0,6 & 13,4 & 28,3 & 31,2 & 31,8 \\
\hline 2 & 50,4 & 23,6 & 0,4 & 12,8 & 27,5 & 30,6 & 31,2 \\
\hline 3 & 42,7 & 31,7 & 0,1 & 12,8 & 27,1 & 29,9 & 30,4 \\
\hline 4 & 52,2 & 21,4 & 0,1 & 12,5 & 26,3 & 29,2 & 29,7 \\
\hline 5 & 42,7 & 30,6 & 2,0 & 11,9 & 25,4 & 29,6 & 30,2 \\
\hline 6 & 57,2 & 18,0 & 0,8 & 11,6 & 24,6 & 29,9 & 29,4 \\
\hline
\end{tabular}


Los clínkeres fueron evaluados en cuanto al "contenido de alita" por espectroscopía infrarroja y por difracción de rayos X. Además, se observaron por microscopía óptica con luz reflejada en un microscopio Metallux 3 y se le determinó el contenido de cal libre a muestras calcinadas a distintas temperaturas.

A los cementos elaborados a partir de ellos se les determinó el contenido de agua fija (grado de hidratación), para lo cual se elimina el agua no combinada por un tratamiento con alcohol isopropílico, seguido de acetona y calentamiento a 110 grados celsios. Al producto así obtenido se le determina la pérdida por ignición, que se relaciona con la resistencia a compresión alcanzada por morteros de cemento sin adición, con coeficientes de correlación mayor de 0,90 [11].

\section{RESULTADOS}

Con los valores de fase líquida que se muestran en la tabla 3, y aplicando el diseño propuesto, se obtienen las ecuaciones siguientes:
The "alite content" was evaluated by X ray diffraction [9] and by infrared espectroscopy [8].

These sample were also studied by optical microscopy with a Metallux 3 microscope and their free lime was chemically determined.

Cements were elaborated with this clinkers and were used in fixed water (degree of hydration) determination [11]. For this the noncombined water is eliminated by treatment with isopropyl alcohol followed of acetone and heating at 110 degrees celsius. The product thus obtained is ignited and the lost on ignition is relationed with the compresive strength developed by cement mortar with correlation coeficient greater than 0,90 [11].

\section{RESULTS}

From the values of the liquid phase, and using the proposed model, the next equations were obtained.

$$
\begin{aligned}
& a_{1340}\left(X_{1}, X_{2}\right)=27,3-0,4 X_{1}-0,6 X_{2} \\
& a_{1340}\left(X_{1}, X_{3}\right)=26,4-0,4 X_{1}-1,4 X_{3} \\
& a_{1400}\left(X_{1}, X_{2}\right)=30,2-0,3 X_{1}-0,7 X_{2} \\
& a_{1400}\left(X_{1}, X_{3}\right)=30,1-0,3 X_{1}-0.8 X_{3} \\
& a_{1450}\left(X_{1}, X_{2}\right)=30,8-0,3 X_{1}-0,7 X_{2} \\
& a_{1450}\left(X_{1}, X_{3}\right)=30,6-0,4 X_{1}-0.8 X_{3}
\end{aligned}
$$

Donde $a_{y}$ es la cantidad de fase líquida en $\%$ a la temperatura de " $y$ " grados celsios; $X_{1}$ es el valor codificado para el factor de saturación de cal; $X_{2}$ es el valor codificado para el módulo de sílice; $X_{3}$ es el valor codificado para el módulo de alúmina.

Utilizando las ecuaciones propuestas por Henning [8] y los máximos de difracción propuestos por Yamaguchi et al [9], se estimó el "contenido de alita", mostrándose los resultados en la tabla $4 \mathrm{y}$, al aplicar el diseño propuesto a los valores obtenidos, se encontró:

- Por el método infrarrojo:
Where $a_{y}$ is the quantity of liquid phase in $\%$ at the temperature of " $y$ " celsius degrees.

$X_{1}$ is the coded value for $L S F$.

$X_{2}$ is the coded value for Ms.

$X_{3}$ is the coded value for Ma.

Using the equations proposed by Henning [8] and the maximum of diffraction proposed by Yamaguchi [9], "alite content" was estimated, the results are shown in table 4.

With this values the next equations were calculated:

- Using infrared method:

$$
\begin{aligned}
& Y=2615+1415 X_{1}-419 X_{1} X_{2} \\
& Y=2245+1105 X_{1}-684 X_{3}
\end{aligned}
$$


- Por difracción de rayos $X$ : - Using $X$ ray diffraction method:

$$
\begin{aligned}
& Y=22,25+3,23 X_{1}-1,79 X_{1} X_{2} \\
& Y=21,4+1,2 X_{1}-0,9 X_{3}
\end{aligned}
$$

TABLA 4 TABLE 4

Estimación del contenido de alita por las distintas técnicas

(Estimation of "alite content" by different technics)

\begin{tabular}{|c|c|c|}
\hline Muestra (Sample) & DRX & IR \\
\hline 1 & 21,0 & 1.085 \\
2 & 23,5 & 4.773 \\
3 & 21,9 & 1.310 \\
4 & 22,6 & 3.293 \\
5 & 21,6 & 1.107 \\
6 & 19,4 & 994 \\
\hline
\end{tabular}

Donde DRX es el área del máximo de difracción en centímetros cuadrados $\theta$ IR es el valor obtenido de las ecuaciones de regresión propuestas por Henning utilizando espectroscopía infrarroja.

Donde $Y$ es el "contenido relativo de alita"; $X_{1}$, $X_{2}$ y $X_{3}$ toman, respectivamente, el mismo significado que en el caso anterior.

Como se puede observar, las ecuaciones obtenidas por ambos métodos son similares en cuanto a su forma general.

Al carecer de patrones adecuados, no pudieron calibrarse los equipos, motivando que las ecuaciones sólo sean indicativas de la variación del contenido de alita.

Los resultados del contenido de cal libre, de las muestras calcinadas a distintas temperaturas, se muestran en el tabla 5.

Con esos valores, y aplicando el diseño propuesto, se calcularon las ecuaciones siguientes:
Where $Y$ is "alite content" $X_{1}, X_{2}$ and $X_{3}$ take respectively the coded values of LSF, Ms and Ma.

As it could be seen this equations are of a similar form.

The equipment could not be calibrated because of the lack of standards. Making this equations only indicative of the variation of "alite content".

The free lime of the samples ignited at different temperatures are shown in table 5.

with this values the next equations were calculated.

$$
\begin{aligned}
& \% \mathrm{Cl}_{950}\left(X_{1}, X_{2}\right)=21,6+1,8 X_{1}+0,8 X_{1} X_{2} \\
& \% \mathrm{Cl}_{950}\left(X_{1}, X_{3}\right)=21,5+0,7 X_{1}-0,3 X_{3}+0,8 X_{1} X_{3} \\
& \% \mathrm{Cl}_{1200}\left(X_{1}, X_{2}\right)=12,0+X_{1}-1,1 X_{2}-0,8 X_{1} X_{2} \\
& \% \mathrm{Cl}_{1200}\left(X_{1}, X_{3}\right)=12,3+0,6 X_{1}-0,9 X_{3}+0,8 X_{1} X_{2} \\
& \% \mathrm{Cl}_{1300}\left(X_{1}, X_{2}\right)=1,2+0,4 X_{2}+0,3 X_{1} X_{2} \\
& \% C_{1300}\left(X_{1}, X_{3}\right)=1,5+0,4 X_{1} X_{3} \\
& \% C l_{1400}\left(X_{1}, X_{2}\right)=1,1+0,2 X_{2} \\
& \% C l_{1400}\left(X_{1}, X_{3}\right)=1,1+0,1 X_{3}
\end{aligned}
$$


TABLA 5 TABLE 5

Contenido de cal libre de las muestras calcinadas a distintas temperaturas

Fre lime contents of samples ignited at different temperatures

\begin{tabular}{|c|c|c|c|c|c|c|}
\hline \multirow{2}{*}{$\begin{array}{c}\text { Temperatura } \\
\text { calclinacín } \\
\text { (lgnitlon } \\
\text { temperature) }\end{array}$} & $\mathbf{1}$ & $\mathbf{2}$ & $\mathbf{3}$ & $\mathbf{4}$ & $\mathbf{5}$ & $\mathbf{6}$ \\
\cline { 2 - 7 } & $\mathbf{1}$ & 22,85 & 23,87 & 18,72 & 20,85 & 21,53 \\
\hline 950 & 20,84 & 17,73 & 14,14 & 14,75 & 15,00 & 14,36 \\
1.100 & 16,18 & 14,98 & 10,72 & 11,14 & 12,09 & 10,71 \\
1.200 & 11,31 & 12,38 & 10,55 & 9,29 & 10,43 & 10,52 \\
1.220 & 10,86 & 10,78 & 5,87 & 6,37 & 6,04 & 5,90 \\
1.250 & 5,40 & 2,23 & 1,00 & 0,76 & 1,61 & 1,02 \\
1.300 & 1,11 & 2,17 & 0,79 & 0,67 & 0,57 & 1,06 \\
1.350 & 1,20 & 0,91 & 1,17 & 1,47 & 1,31 & 1,11 \\
1.400 & 0,94 & 0 &
\end{tabular}

Donde $\% \mathrm{Cl}_{y}$ es el contenido de cal libre que se obtiene en una muestra calcinada a la temperatura de "y" grados celsios y las demás variables toman los valores de los casos anteriores.

Por otro lado se obtuvieron clínkeres calcinando gradualmente las muestras desde temperatura ambiente hasta 1.400 grados celsios, con los cuales se elaboraron cementos cuyas composiciones químicas se muestran en la tabla 6 , y se les determinó el grado de hidratación [11] y la resistencia a compresión se calculó a partir de ésta, mostrándose los valores en las tablas 7 y 8 respectivamente.

Al procesar estos valores, se obtuvieron las ecuaciones siguientes:
Where $\% \mathrm{Cl}_{y}$ is the free lime contents in the ignited samples at " $y$ " celsius degrees, the other variables take the same value of the foregoing equations.

With the clinkers obtained igniting the samples gradually from ambient temperature to 1.400 celsius degrees, cements which composition are shown in table 6 were produced.

Using the method proposed in [11] degree of hydration and compressive stregth were determined and calculated respectively. The results are shown in tables 7 and 8.

The processing of this values gave the next equations:

$$
\begin{aligned}
& R_{24 \mathrm{~h}}\left(X_{1}, X_{2}\right)=24+0,2 X_{1}-0,6 X_{2}-1,3 X_{1} X_{2} \\
& R_{24 \mathrm{~b}}\left(X_{1}, X_{3}\right)=23+1,2 X_{1}-1,6 X_{3}-1,0 X_{1} X_{3} \\
& R_{3 d}\left(X_{1}, X_{2}\right)=31,1-0,3 X_{1}-0,2 X_{2}-0,6 X_{1} X_{2} \\
& R_{3 d}\left(X_{1}, X_{3}\right)=30,9+X_{1}-1,3 X_{1} X_{3} \\
& R_{7 d}\left(X_{1}, X_{2}\right)=35,6-1,9 X_{2}-0,3 X_{1} X_{2} \\
& R_{7 d}\left(X_{1}, X_{3}\right)=38,8+1,2 X_{1}+X_{3}-0,9 X_{1} X_{3} \\
& R_{28 d}\left(X_{1}, X_{2}\right)=44,9-0,4 X_{1} X_{2} \\
& R_{28 d}\left(X_{1}, X_{3}\right)=45+0,32 X_{1}
\end{aligned}
$$

Donde $\mathrm{R}_{\mathrm{y}} \mathrm{d}$ es la resistencia a compresión a la

Where $R_{y} d$ is the compressive stregth at the 
edad de "y" horas (o días) de curado y las demás variables toman los valores de los casos anteriores. curing age of " $y$ " days or hours.

$X_{1}, X_{2}$ and $X_{3}$ take respectively the coded values of LSF, MS and Ma.

TABLA 6 TABLE 6

Composición química de los diferentes cementos (Chemical composition of different cements)

\begin{tabular}{|c|c|c|c|c|c|c|c|c|c|}
\hline \multirow{2}{*}{$\begin{array}{c}\text { Muestra } \\
\text { (Sample) }\end{array}$} & \multicolumn{9}{|c|}{ Contenldos en \% en masa (Contents in mass \%) } \\
\hline & $\mathrm{SiO}_{2}$ & $\mathrm{Al}_{2} \mathrm{O}_{3}$ & $\mathrm{Fe}_{2} \mathrm{O}_{3}$ & $\mathrm{CaO}$ & $\mathrm{MgO}$ & PPI & $\mathrm{SO}_{3}$ & $\mathbf{R I}$ & CL \\
\hline 1 & 20,5 & 6,2 & 3,9 & 61,2 & 1,0 & 1,2 & 3,4 & 0,7 & 0,51 \\
\hline 2 & 19,6 & 5,9 & 3,9 & 61,9 & 0,6 & 1,3 & 3,5 & 0,6 & 1,13 \\
\hline 3 & 20,6 & 5,8 & 3,8 & 61,3 & 1,0 & 1,2 & 3,2 & 1,1 & 0,64 \\
\hline 4 & 20,2 & 5,6 & 3,8 & 61,6 & 0,8 & 1,8 & 3,2 & 1,9 & 0,55 \\
\hline 5 & 20,8 & 6,2 & 3,6 & 60,9 & 0,9 & 1,6 & 3,2 & 1,0 & 0,64 \\
\hline 6 & 19,7 & 5,9 & 3,4 & 62,5 & 0,7 & 1,5 & 3,4 & 1,0 & 0,56 \\
\hline
\end{tabular}

TABLA 7 TABLE 7

Resultados del grado de hidratación a las distintas edades (Degree of hydration results at different ages)

\begin{tabular}{|c|c|c|c|c|}
\hline \multirow{2}{*}{$\begin{array}{c}\text { Muestra } \\
\text { (Sample) }\end{array}$} & \multicolumn{3}{|c|}{ Grado de hidratación a la edad de (Degreeof hydration at the curing age of) } \\
\cline { 2 - 5 } & $\mathbf{2 4 h}$ & 3d & $\mathbf{7 d}$ & $\mathbf{2 8 d}$ \\
\hline 1 & 12,23 & 15,22 & 17,47 & 20,51 \\
2 & 14,05 & 15,81 & 17,70 & 21,29 \\
4 & 12,98 & 15,71 & 16,46 & 21,51 \\
5 & 12,31 & 15,52 & 16,28 & 20,63 \\
\hline
\end{tabular}

TABLA 8 TABLE 8

Resultados de resistencia a compresión calculados (Calculated compressive strength results)

\begin{tabular}{|c|c|c|c|c|}
\hline \multirow{2}{*}{$\begin{array}{c}\text { Muestra } \\
\text { (Sample) }\end{array}$} & \multicolumn{4}{|c|}{$\begin{array}{c}\text { Resistencia a compresión estimada en MPa a la edad de: } \\
\text { (Calculated compressive strength in MPa at the curing) }\end{array}$} \\
\cline { 2 - 5 } & $24 h$ & 3d & 7d & 28d \\
\hline 1 & 22,6 & 30,0 & 37,2 & 44,4 \\
2 & 26,6 & 31,9 & 37,9 & 45,2 \\
4 & 24,0 & 31,6 & 34,0 & 44,3 \\
6 & 22,8 & 31,0 & 33,4 & 45,0 \\
\hline
\end{tabular}




\section{DISCUSIÓN DE LOS RESULTADOS}

La reactividad se mide por el contenido de cal libre [2, 3], encontrándose que el mismo disminuye linealmente con el incremento de temperatura (tabla 9).

Al incrementarse el valor de cualquiera de las tres variables estudiadas (FSC, Ms y Ma), disminuye el contenido de fase líquida.

\section{DISCUSSION AND COMMENTS}

The increment of either of the variables studied diminishes the liquid phase content.

Reactivity is measured by the free lime content [2, 3] and this content diminishes linearly with temperature increments see table 9.

Tabla 9 Table 9

Parámetros de regresión entre el contenido de cal libre y la temperatura

(Regression parameters between free lime content and temperature)

\begin{tabular}{|c|c|c|c|}
\hline Muestra (Sample) & Const. & Pendiente (Slope) & Coef. correl. (Correl. coef.) \\
\hline 1 & 70,3 & $-0,051$ & $-0,9621$ \\
2 & 76,1 & $-0,054$ & $-0,9536$ \\
3 & 75,1 & $-0,054$ & $-0,9731$ \\
4 & 63,1 & $-0,045$ & $-0,9549$ \\
5 & 68,9 & $-0,050$ & $-0,9644$ \\
6 & 69,5 & $-0,050$ & $-0,9705$ \\
\hline
\end{tabular}

Analizando todo lo anterior y teniendo en cuenta las ecuaciones que dan el contenido de cal libre, en función de los parámetros estudiados, a las temperaturas de 1.300 a 1.400 grados celsios puede recomendarse trabajar con valores bajos de ambos módulos y valor alto del FSC, con lo que debiera obtenerse una mayor reactividad.

Al disminuir el módulo de alúmina (dentro del rango estudiado), aumenta el contenido de fase líquida [12]; esto se confirma por las observaciones microscópicas, como se muestra en las fotos 1 y 2 .

Al aumentar el factor de saturación de cal se observa mayor cantidad de alita (fotos 1 y 3 ), disminuyendo además el contenido de fase líquida.

El aumento del módulo de sílice genera nidos de belita beta y la disminución de la fase líquida (foto 4). Esto se corroboró por difracción de rayos $X$ ya que la belita beta incrementó a un $140 \%$ su área.

Tanto en facilidad de quema de crudos, como en reactividad del cemento, se ha detectado como significativa la interacción con signo negativo entre el factor de saturación de cal y el módulo de sílice, esto sugiere trabajar con
Analyzing the facts presented so far, working with high values of $L S F$ and low values off $M s$ and Ma for obtaining the best reactivity is proposed.

When $\mathrm{Ma}$ is diminished (in the studied range), the liquid phase is enhanced [12] and this is confirmed in pictures 1 and 2 taken by microscopy.

When LSF is incremented alite quantity is enhanced and liquid phase is diminished (pictures 1 and 3).

Increments in Ms produce beta belita nests and less liquid phase (picture 4). The belite increment was confirmed by $X$ ray diffraction, showing and increment of $140 \%$ of the area of the maximum of diffraction of beta belita.

In raw mix burnability and in cement reactivity a negative interaction between LSF and Ms could be seen. Working at high level of LSF and low Ms is suggested. 
factor de saturación alto y módulo de sílice bajo.

El incremento del módulo de alúmina hace disminuir el "contenido de alita" (ecuaciones 8 y 10), ya que el $C_{3} A$ tiene un contenido específico de óxido de calcio mayor que el $\mathrm{C}_{4} \mathrm{AF}[12]$.

Aunque los valores de resistencia a compresión calculados [11] muestran que con módulos de alúmina altos se obtienen valores mayores de resistencia a compresión calculados a edades mayores de 24 horas, y menores a esta edad, donde la resistencia menor se alcanza con módulo de alúmina bajo, debe aclararse que las diferencias observadas no son estadísticamente significativas desde el punto de vista del ensayo de resistencia.

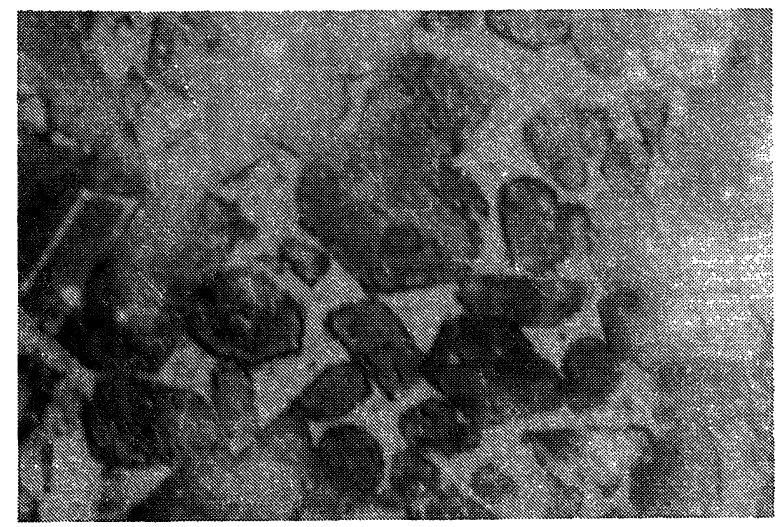

Foto 1.-Nital al $1 \% 1.000 x$. Muestra 2, clínker del laboratorio calcinado a 1.400 grados celsios. Abundante fase líquida y alita idiomorfa.

Picture 1.-Nital at $1 \% 1.000 x$. Sample 2, Laboratory clinker ignited at 1.400 celsius degrees. Abundant liquid phase and idiomorfic alite.

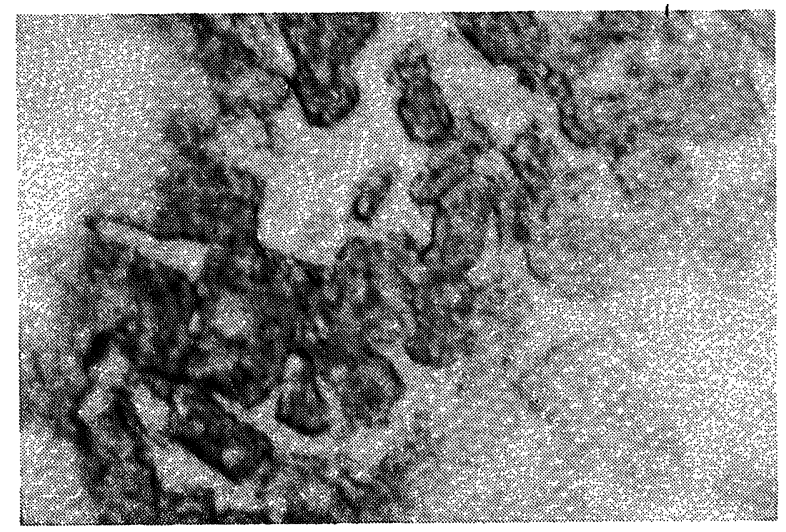

Foto 2.-Nital al $1 \%$ 1.000x. Muestra 5, clínker de laboratorio calcinado a 1.400 grados celsios. Disminución de fase líquida, aumento de porosidad, alita con bordes corroidos, matriz diferenciada.

Picture 2.-Nital at $1 \%$ 1.000x. Sample 5, Laboratory clinker ignited at 1.400 celsius degrees. Decrease of liquid phase, porosity increase, alite with corroded edges, diferentiated matrix.
Enhancing Ma diminishes alite contents (equations 8 and 10).

This is due to the higher specific contents of calcium oxide in tricalcium aluminate than in tetracalciumaluimnoferrite [12].

The calculated compressive stregth values [11], show that high Ma enhances stregth at all ages but at 24 hours a lower Ma gives the higher strength. But the differences in compressive strength observed are not statistically significant.

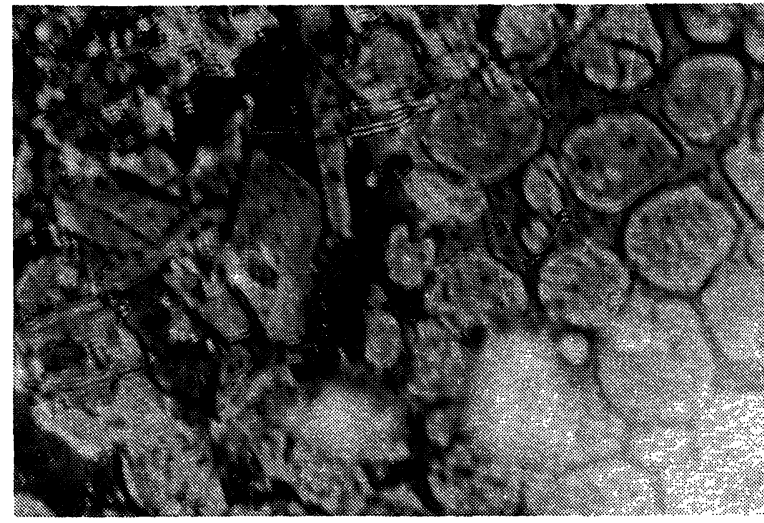

Foto 3.-Nital al $1 \% 1.000 x$. Muestra 3, clinker de laboratorio calcinado a 1.400 grados celsios. Campo de alitas y belitas con poca fase líquida.

Picture 3.-Nital at $1 \% 1.000 x$. Sample 3, Laboratory clinker ignited at 1.400 celsius degrees. Alites and belites with little liquid phase.

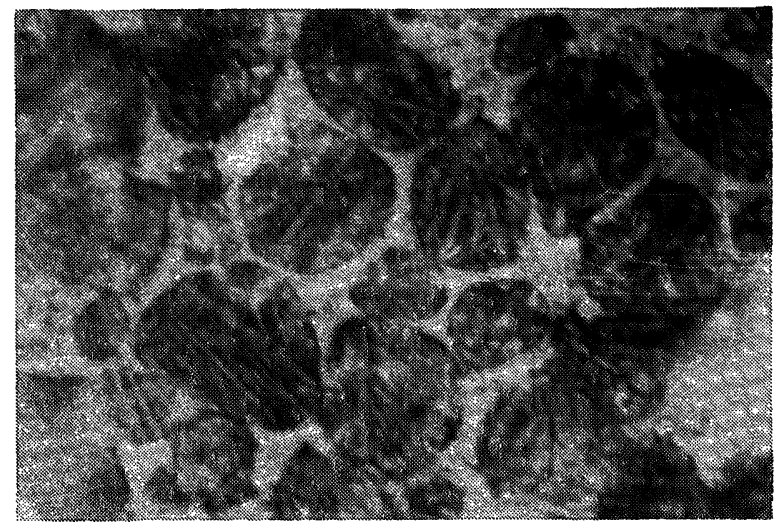

Foto 4.-Nital al $1 \%$ 1.000x. Muestra 3, clinker de laboratorio calcinado a 1.400 grados celsios. Campo de belitas beta.

Picture 4.-Nital at $1 \% 1.000 x$. Sample 3, Laboratory clinker ignited at 1.400 celsius degrees beta belites. 


\section{CONCLUSIONES}

Las técnicas de diseño de experimento permiten investigar la facilidad de quema de crudos de cemento, obteniendo resultados que corroboran la teoría y confirman los resultados de las técnicas instrumentales. Esto permite un ahorro sustancial de tiempo, recursos y costos en la investigación.

La mejor facilidad de quema del crudo y la mayor reactividad del cemento se obtienen trabajando con FSC alto $(0,95)$, Ms bajo $(2,0)$ y Ma bajo $(1,56)$.

Se encontró una interacción con signo negativo tanto en facilidad de quema como en reactividad del cemento entre el FSC y el Ms.

Para el rango de concentraciones estudiado disminuye el contenido de fase líquida al aumentar cualquiera de los tres parámetros.

Se recomienda realizar un estudio en condiciones industriales de tal forma que se acerquen gradualmente al nivel propuesto de los parámetros, teniendo en cuenta que sólo en las condiciones de trabajo del horno se podrá determinar el óptimo.

\section{CONCLUSIONS}

Experimental design technics enables investigation of cement raw mix burnability, obtaining results that corroborate theory and instrumental technics results. This produces great savings in time, resources and investigation costs.

Working at high $\operatorname{LSF}(0,95)$ and low values of Ms $(2,0)$ and $M a(1,56)$ gives the best reactivity of the raw mix.

When any of the three parameters are enhanced within the studied range of concentrations, the liquid phase diminishes. The working at low Ma should be proposed.

A new study in industrial conditions is proposed in order to change the target gradually in the direction where the best results were obtained, considering that only in the kiln working conditions a real optimum could be obtained.

\section{BIBLIOGRAFIA}

[1] BORSCHEIN G. et al: "Necesidades energéticas en la industria del Cemento". Publicaciones de investigación, Instituto de cemento de Dessau. R.D.A. (1982) pp. 13.

[2] KOCK $\mathrm{H}$. et al: "A statistical model for determination of burnability of raw mixes". VI Simposio de la quimica del cemento, Moscú, 1974.

[3] FUNDALL E.: "The burnability of cement raw mixes". World Cement Technology vol. 10 \# 6, (1979) pp. 195.

[4] TIMASHEV V. V.: "La cinética de formación del clínker. La estructura y composición del clínker y sus fases". VII Simposio de la química del cemento, París, 1980

[5] RIVERA M. et al: "Formation of Portland clinker. Studies on sinthetic raw mixes". Advances in cement research Vol. 1 \# 1, oct. 1987 pp. 52.

[6] PUERTAS F. et al: "Reactividad y facilidad de quema de crudos de cemento hechos con escorias de altos hornos cristaslizadas, Parte 1". Zement Kalk Gips vol. 41 \# 8 (1988) pp. 398.

[7] DUQUE G. et al: "Evaluación de la reactividad de quema del crudo de la empresa "Rene Arcay". Ingenieria Estructural \# 1 (1989) pp. 5.

[8] HENNING O. et al: "Spectroscopic method for direct determination of mineral composition of clinkers" Silicattechnique vol. 20 pp. 54 (1969) y vol. 23 pp. 24 (1972).

[9] YAMAGUCHI G. et al: "Analysis of portland cement clinker" V Simposio de la química del cemento Tokio (1968) tomo 1 pp. 181.

[10] LÓPEZ PLANES R.: "Diseño estadístico de experimentos”. Editorial científica técnica. La Habana (1988) pp. 120.

[11] DUQUE G. et al: "Determinación del agua fija como método de control de resistencia a compresión en morteros de cemento sin adición". Ingeniería Estructural, \# 2 (1989) Pend. Public.

[12] LEA F. M.: "La química del cemento y el hormigón”. tercera edición Edward Arnold (publishers) Ltd. (Glasgow), Gran Bretaña. 